\title{
IDENTIDAD ÉTNICA MAPUCHE E IMAGINARIOS SOCIALES DEL BIENESTAR EN LA REGIÓN DEL BIOBÍO, CHIILE
}

\author{
IDENTIDADE ÉTNICA MAPUCHE E IMAGINÁRIOS SOCIAIS DE BEM- \\ ESTAR NA REGIÃO DO BIOBÍO, CHILE \\ MAPUCHE ETHNIC IDENTITY AND SOCIAL IMAGINARIES OF \\ WELL-BEING IN BIOBÍO REGION, CHILE
}

\author{
Andrea Aravena Reyes ${ }^{1}$, Claudia Cerda Zúñiga ${ }^{1}$, Pablo Alcota \\ Poblete $^{1}$ e Natalia Zañartu Canihuante ${ }^{2}$ \\ ${ }^{1}$ Universidad de Concepción, Concepción, Chile \\ ${ }^{2}$ Universidad del Bío-Bío, Chillán, Chile \\ ${ }^{3}$ Universidad San Sebastián, Concepción, Chile
}

\begin{abstract}
RESUMEN: Analizamos los imaginarios sociales del bienestar subjetivo en personas que se auto identifican como mapuche en el Gran Concepción, Biobío, Chile. El bienestar subjetivo otorga importancia a aquellos factores que los sujetos consideran como un aporte positivo en su vida personal, familiar o comunitaria, que generan percepciones de satisfacción y felicidad. Esto también forma parte de lo que se considera como parte constitutiva del buen vivir de los pueblos originarios. Se trabajó con un enfoque cualitativo, a través de entrevistas semi-estructuradas. Los resultados indican que los imaginarios sociales del bienestar subjetivo son diversos y variados dependiendo de los niveles en los cuales la identidad es vivida: individual, colectivo o estructural. Todavía, los resultados indican que esos imaginarios se construyen en torno a la resignificación de la identidad individual y colectiva, como parte de un proceso de relación entre sujetos, entorno y/o naturaleza, donde confluyen percepciones, representaciones, valoraciones y emociones asociadas no solo a la felicidad, la seguridad y el respeto, sino también a la trascendencia como pueblo portador de una identidad Mapuche diferenciada.
\end{abstract}

PALABRAS CLAVES: Bienestar; Identidad étnica; Imaginarios sociales; Mapuche.

RESUMO: Analisamos os imaginários sociais do bem-estar subjetivo em pessoas que se identificam como mapuche, no Gran Concepción, Biobío, Chile. O bem-estar subjetivo outorga importância a aqueles fatores que o sujeito considera um aporte positivo em sua vida pessoal, família ou comunitária, e que geram percepções de satisfação e felicidade. Este também é parte constitutiva do bom viver dos povos originários. Se trabalhou com um enfoque qualitativo, através de entrevistas semi-estruturadas. Os resultados indicam que os imaginários sociais do bem estar subjetivo são diversos e variados dependendo dos níveis os quais a identidade é vivida: individual, coletivo ou estrutural. Ainda, que são construídos em torno da ressignificação da identidade individual e coletiva, como parte de um processo de relacionamento entre sujeitos, ambiente e/ou natureza, onde convergem percepções, representações, apreciações e emoções associadas não só à felicidade, à segurança e ao respeito, mas também à transcendência como um povo portador de uma identidade diferenciada mapuche.

PALAVRAS CHAVE: Bem-estar; Identidade étnica; Imaginários sociais; Mapuche.

ABSTRACT: We analyze the social imaginaries of the subjective well-being in people who identify as Mapuche in Greater Concepción, Chile. Subjective well-being gives importance to those factors that the subject considers to be a positive contribution to their personal, family or community life, and that generate perceptions of satisfaction and happiness. This also constitutes part of what is perceived as a good life for native people. The work considered a qualitative approach through semi-structured interviews. Results indicate that the social imaginaries of subjective well-being are diverse and varied, depending on the levels at which the identity is lived: individual, collective or structural. Furthermore, the results indicate that the social imaginaries of the subjective welfare are built around the resignification of individual and collective identity, as part of a process of relationship between subjects, environment and/or nature, in which they converge perceptions, representations, values and emotions associated not only to happiness, security and respect, but also to transcendence as a people carrying a differentiated Mapuche identity.

KEYWORDS: Well-being; Ethnic identity; Social imaginaries; Mapuche. 


\section{Introducción}

En Chile, la población mapuche bordea el millón quinientas mil personas, lo que corresponde a un $9.1 \%$ de la población nacional. En su mayoría, se localiza en zonas urbanas, concentrando su ubicación en Santiago (Región Metropolitana), Temuco (Región de la Araucanía) y Concepción (Región del Biobío). En la Región del Biobío la población que adscribe al pueblo mapuche alcanza a 104.582 personas, habitando el $78 \%$ de ellas en zonas urbanas (Ministerio de Desarrollo Social, 2015).

Esta región es reconocida como la frontera entre el territorio mapuche y los españoles, primero y los mapuche y el Estado de Chile después, - en honor al río Biobío que marcó dicha frontera hacia fines del período de la Conquista española (1536-1598) (Goicovich, 2007) y la consolidación de una suerte de soberanía territorial y jurisdicción mapuche a partir del Tratado de Quilin en 1641 (Ortiz, 2015). Durante la llamada Guerra de Arauco, que se extendió entre los siglos XVI y XVII sin que exista a la fecha consenso respecto de sus inicios y términos exactos, el pueblo mapuche sufrió la disputa de su territorio ocurriendo sucesivas incursiones militares del ejército español (Bengoa, 1990).

La Independencia de Chile (1818) no significó el fin de esta guerra. Por el contrario ella se intensificó con la ocupación militar de sus tierras por el Estado chileno durante la llamada "Pacificación de la Araucanía" (1860-1883) (Bengoa, 1985), período en que comienza el establecimiento de la propiedad indígena en reducciones y que se extiende hasta 1927 (González, 1986). Con posterioridad, se implementa la "división" de dichas reducciones y el establecimiento de la propiedad privada en tierras otrora comunitarias, situación que persiste hasta la dictadura militar (1973-1990) (Bengoa, 1990).

Con el retorno a la democracia en Chile en 1990, se implementa la llamada Nueva Ley Indígena (1993) que, a pesar de haber sido trabajada de manera participativa con las organizaciones indígenas del país no ha logrado revertir drásticamente el despojo territorial mapuche (Boccara \& Seguel-Boccara, 1999). Por el contrario, se ha sostenido que en el marco de una política de "neoliberalismo multicultural" (Bolados, 2012), el despojo de las tierras mapuche, el cambio del uso del suelo y la expoliación de su entorno en manos de empresas forestales e hidroeléctricas se ha potenciado. Desde las organizaciones mapuche ello ha sido denunciado como un daño irreparable a su ecosistema y cultura, reivindicando su derecho a la autonomía y a la autodeterminación (Pairican, 2014; Calbucura, 2006) y movilizando a sus comunidades, organizaciones y dirigentes.

El Estado ha respondido ejerciendo violencia policial, militarizando las comunidades mapuche en conflicto - principalmente con empresas forestales - encarcelando, persiguiendo y desde el año 2000 aplicando la Ley Antiterrorista de seguridad interior (INDH, 2017). Por otra parte, la migración mapuche a las ciudades, documentada desde 1930, se acentúa hacia fines de 1980 y se visibiliza a partir del Censo de Población de 1992 (Aravena, 1995). Alguna de sus causas son la pérdida y degradación de sus territorios, la falta de oportunidades laborales y la demanda de mano de obra proveniente de las ciudades, dando inicio a la salida de jóvenes para ayudar a la subsistencia familiar. Las migraciones implican procesos de adaptación a los medios urbanos, donde los emigrados de comunidades sufren discri- 
minación, confirmando, negando o asimilando su identidad en un nuevo entorno social y ambiental (Aravena, 2001). Conforme emana de este estudio, las vivencias y percepciones subjetivas sobre dichos procesos se han traspasado de generación en generación generando efectos aún no dimensionados en el bienestar subjetivo de los mapuche ${ }^{1}$ en la actualidad.

Bajo el contexto consignado emerge el objetivo del presente estudio, que es analizar los imaginarios sociales del bienestar en personas mapuche del Gran Concepción. Para cumplir con este objetivo se realizaron 58 entrevistas semi-estructuradas, obteniéndose información que fue audio grabada y luego transcrita, para posteriormente realizar análisis hermenéutico y de discurso. A continuación, se señalan antecedentes teóricos del presente estudio, para posteriormente encontrar el apartado del método, resultados, discusión y conclusiones.

\section{Antecedentes teóricos}

\section{Identidad e identidad étnica}

Las definiciones de identidad varían de acuerdo a los diferentes aspectos considerados por cada una de ellas, transitando desde factores biológicos a sociales, históricos y culturales, considerando aspectos de naturaleza individual, como elementos intrapsíquicos, cognitivos, emocionales y conductuales, a aspectos de naturaleza colectiva y relacional (Erickson, 1971; Ibáñez, 2001, 2003; Mujica, 2007; Tajfel, 1981; Turner, 1985; Vygotsky, 1996; Aravena \& Cerda, 2018). La identidad social se configura de manera subjetiva y contextual y, de acuerdo a Tajfel (1981), corresponde a "aquella parte del autoconcepto de un individuo que deriva del conocimiento de su pertenencia a un grupo social, junto con el significado valorativo y emocional asociado a dicha pertenencia” (Tajfel, 1981, p. 292). Además, la identidad correspondería al resultado de una historia que se construye (Berger \& Luckmann, 1968) y contada, a partir de la acción y el intercambio comunitario (Gergen, 1996) y se enmarca en una realidad representada (Elcheroth, Doise, \& Reicher, 2011), instituida e imaginada (Castoriadis, 2007).

La identidad étnica es un tipo de identidad social que supone aspectos o identificaciones individuales, históricas y colectivas a un pueblo o cultura, y se compone de la identificación al colectivo, en un conjunto de relaciones de alteridad. Este sentido de pertenencia se modifica a lo largo del desarrollo humano debido a procesos de aprendizaje, indagación y compromiso (Phinney \& Ong, 2007). La identidad étnica ha sido estudiada como una "construcción ideológica histórica, contingente, relacional, no esencial y eventualmente variable, que manifiesta un carácter procesual y dinámico, y que requiere de referentes culturales para constituirse como tal y enfatizar su singularidad" (Bartolomé, 2008, p. 83). Corresponde a una "forma de ser" que es en sí, una forma diferenciadora de los demás (Mujica, 2007; Paramo, 2008). En Chile, la identidad étnica ha sido estudiada, observada y analizada en distintos niveles que corresponden a diferentes planos de la realidad social y de las vivencias de la identidad (Aravena, 2006): (a) a nivel micro social o individual, la identidad étnica refiere a la conciencia de pertenencia que experimentan los individuos respecto de su adscripción identitaria y a las prácticas individuales que refuerzan dicho sentimiento de pertenencia; (b) a nivel meso social o grupal, la identidad étnica se vincula 
a la movilización de la misma en términos colectivos, para efectos de su reivindicación, asociatividad, establecimiento de fronteras entre grupos y prácticas distintivas, y; (c) a nivel macro social o estructural, la identidad étnica resulta modelada por determinantes de naturaleza ideológica, política, económica y social del entorno (Aravena, 2006).

\section{Identidad étnica e imaginario social}

De acuerdo a Taylor (2006) los imaginarios sociales son los modos en que las personas imaginan su existencia social y su entorno, "siendo una concepción colectiva que hace posible las prácticas comunes y un sentimiento ampliamente compartido de legitimidad" (Taylor, 2006, p. 37). Esta concepción es compartida en un lugar y tiempo determinados, y es influenciada por la historia y posición del sujeto en la sociedad. De acuerdo a Baeza (2015) los imaginarios sociales son "una manera compartida por grupos de personas de representarse en el espacio y el tiempo" (Baeza, 2000, p. 9), siendo "construcciones sociales que permiten significar el mundo en el que nos desenvolvemos" (Baeza, 2015, p. 132). Los imaginarios sociales también pueden ser expresados como un sentimiento colectivo de las poblaciones humanas a través de la identidad y de la memoria colectiva (Aravena \& Baeza, 2017). Por otro lado, los imaginarios sociales no son construcciones estáticas, sino que son capaces de subvertir la realidad institucionalizada, siendo una importante fuente de posibilidades alternativas a la realidad socialmente dominante (Carretero, 2001). Por lo tanto, es posible reconstruirlos o hacer surgir nuevos imaginarios sociales a través de la acción social.

En relación a la identidad étnica, existen imaginarios sociales que pueden ser propios o atribuidos, positivos o negativos, impuestos, naturalizados y/o hegemónicos. Para comprender cómo se construyen estos imaginarios, resulta necesario indagar en tres posicionamientos que clarifican los procesos de identificación de las personas/colectividades con una determinada identidad étnica: uno espacial que involucra la apropiación colectiva de un espacio físico o simbólico; uno temporal referente a la apropiación del tiempo pasado (historia) - presente y futuro (utopía) y uno relacional que implica la manera en que el "Otro" es percibido desde el "Nosotros" (Baeza, 2015, p. 134). Analizando estos posicionamientos, se puede comprender la definición de identidades colectivas, y como es en el caso de este estudio, ver la manera en que se construyen los imaginarios sociales en torno a la idea de bienestar, asociado a las identidades de los pueblos originarios.

\section{Elementos que conforman los imaginarios de la identidad mapuche en la ciudad}

Los estudios sobre autodefinición étnica mapuche en Chile, en adultos y jóvenes, destacan aspectos objetivos, subjetivos e inter-relacionales en torno a la construcción de dicha identidad. Estos se distribuyen en los diferentes planos o niveles micro, meso y macro social, descritos con anterioridad.

Dentro de los componentes objetivos de auto identificación del "Ser-Mapuche", en términos individuales y colectivos se considera la filiación, entendida como el vínculo consanguíneo o küpal, que se traspasa a través del apellido, así como el territorio de procedencia o tuwün (Merino \& Tocornal, 2012), que refiere a los vínculos familiares de origen territorial. También se ha descrito como auto adscriptiva la condición de ser hablante de mapudungun [idioma mapuche] (Merino, Quilaqueo, \& Sáiz, 2008), como elemento que 
además valida la identificación con el pueblo. A nivel colectivo o meso social, la participación en ritos o ceremonias evidencia el vínculo comunitario que se tiene tanto con la familia como con la comunidad (Cuminao y Moreno, como se citó en Antileo, 2010; Merino \& Toconal, 2012). Ello también se manifiesta en la participación en asociaciones y organizaciones urbanas (Aravena, 2002). Por otro lado, a nivel macro social o estructural, se habla del aspecto físico como elementos diferenciadores y fuente de discriminación asociados a la adscripción al pueblo mapuche (Aravena, 2001, 2003; Merino, 2007).

También, los aspectos llamados subjetivos definen lo que se entendería por "sentirse mapuche" a nivel micro social, ya que las características objetivas mencionadas no serían suficientes para explicar el sentimiento de pertenencia. De esta forma, mientras que "Ser-Mapuche" puede relacionarse con el apellido, "sentirse mapuche" involucra un compromiso emocional, interaccional y social, que se describe como una identidad activa y proactiva, más que instrumental - búsqueda de beneficios -, o pasiva. El posicionamiento emocional de la identidad étnica, está dado no sólo por el "Ser-Mapuche", sino también "sentirse" como tal. Así, mientras las expresiones forman parte del discurso público, los sentimientos formarían parte del discurso privado de la identidad étnica (Merino \& Tocornal, 2012; Verkuyten \& Wolf, 2002).

\section{Bienestar subjetivo y valoración de la identidad étnica}

El bienestar ha sido estudiado desde diferentes marcos interpretativos. En la actualidad, y desde el campo de la psicología, uno de ellos lo hace a partir de la noción de bienestar subjetivo, por el que se entiende "una amplia categoría de fenómenos que incluye las respuestas emocionales de las personas, la satisfacción con los dominios, y los juicios globales sobre la satisfacción con la vida” (Diener, Suh, Lucas, \& Smith, 1999).

El bienestar subjetivo concita gran interés a nivel transdisciplinar mundial (Farías, Orella, \& Pérez, 2015) y en el caso de Chile particularmente ha sido considerado como un tópico especial en el informe de Desarrollo Humano del año 2012 (PNUD, 2012), entendiéndose que puede ampliar o modificar la concepción de desarrollo humano. En dicho informe, el bienestar subjetivo se comprende desde una mirada multidisciplinaria que integra diferentes dimensiones del mismo, tanto a nivel individual como social, aportando la interacción entre ambas dimensiones de la subjetividad. De acuerdo a dicho marco interpretativo, el bienestar subjetivo social se entiende como la valoración que se realiza de los diversos medios que permiten el desarrollo y felicidad de los sujetos, y se construye “a partir de las experiencias negativas o positivas de los individuos en relación con las oportunidades y los soportes que les ofrece el entorno para desarrollar y potenciar una autoimagen satisfactoria" (PNUD, 2012, p. 148). Por lo tanto, el bienestar subjetivo se concreta cuando los individuos se valoran a sí mismos y a la sociedad, de manera positiva.

En los estudios que tratan acerca del bienestar se ha señalado que los juicios, percepciones y emociones que sobresalen en un grupo social, son un importante componente a considerar respecto del bienestar subjetivo de las personas (De Rivera, Kurrien. \& Olsen, 2007; Páez, Martín-Beristain, González, Basabe, \& De Rivera, 2011 ; Páez, Ruiz, Gailly, Kornblit, Wiesenfeld, \& Vidal, 1997). También, hay autores que trabajan con el concepto de clima emocional, para referirse a las emociones que "no son únicamente las vivenciadas por el sujeto, sino aquellas que el sujeto percibe que predominan en su entorno, tanto en sus grupos de pertenencia o endogrupos, como en los exogrupos relevantes" (Páez et al., 
1997, p. 82), la percepción de un clima emocional positivo o negativo, podría incidir en el bienestar de las personas que se identifican con algún pueblo originario. Otros autores han definido bienestar subjetivo como una percepción continua por parte de un individuo, de que su vida es buena y agradable (Myers, 1992, citado en Espinosa \& Tapia, 2011). Así, han relacionado los procesos identitarios-individual y social- a la vivencia de un bienestar subjetivo, sugiriendo que la identidad "provee de una experiencia psicológica que promueve su ajuste social y su bienestar" (Espinosa \& Tapia, 2011, p.73). Por ello, se ha planteado que tanto la identidad colectiva como la adaptación al medio y el sentido de integración a la comunidad tiene efectos positivos en el bienestar psicológico y social de las personas. Por lo tanto, el estudio del bienestar social entendido como la "evaluación individual acerca de las propias circunstancias y su relación con el funcionamiento de la sociedad” (Keyes, 1998, citado en Espinosa \& Tapia, 2011 , p.73), aporta al conocimiento de la percepción del individuo respecto a su relación con su entorno social y contextual.

Uno de los mayores desafíos que plantean los estudios acerca del bienestar, especialmente cuando este se relaciona con los procesos identitarios, es su análisis cualitativo, a efectos de considerar la subjetividad de las personas, analizando de manera holística, situada e integral, las distintas dimensiones de la experiencia humana que comprende el bienestar, en términos individuales y colectivos, y considerando aspectos de influencia sociopolítica, cultural, histórica y ambiental, entre otros.

\section{Bienestar y buen vivir en los pueblos originarios}

La noción de "buen vivir" (Hasen, 2012; Larrea, 2010; Vanhulst, 2015), en los pueblos indígenas latinoamericanos, enfatiza fundamentalmente la importancia del contexto y del medio ambiente, junto a la relación de interdependencia positiva que debe generarse entre las personas, comunidad y naturaleza, debiendo construirse una relación equilibrada entre aquellos. Las primeras referencias públicas relativas al concepto de buen vivir aparecen en 1990 en Perú y posteriormente en Ecuador y Bolivia, para adquirir toda su fuerza en la Constitución ecuatoriana de 2008 (Acosta \& Martínez, 2009; Gudynas, 2009), y estas apuntan a cuestionar los sistemas de desarrollo que imperan en la modernidad, en pro de establecer otros que incluyan las cosmovisiones de los pueblos originarios, orientándose a buscar un desarrollo integral para todos, incluida la naturaleza. En este sentido, se cuestiona el rol que juega el Estado en la relación producción y explotación de los recursos renovables y no renovables por parte de los privados en los territorios indígenas bajo su jurisdicción. La noción de "buen vivir" ha recibido aportes provenientes de distintos actores de la sociedad, que coinciden fundamentalmente en buscar una alternativa ante el modelo de desarrollo dominante, el que habría destruido vorazmente la naturaleza y el entorno, impactando el territorio y el ambiente, no solamente en términos físicos, sino que también en sus relaciones simbólicas con los habitantes, alterando, además, las relaciones sociales entre los propios habitantes. En consecuencia, uno de los focos del "buen vivir" se asocia a potenciar tanto la participación comunitaria y el sentido de comunidad (Montero, 2004), como el cuidado y respeto por el medio ambiente, lo que difiere del antropocentrismo e individualismo predominantes en la sociedad actual (Moulian \& Marín, 1998). Relativo a lo anterior, se plantea que el buen vivir se asocia igualmente a una condición de bienestar vinculada al reconocimiento de la comunidad de interacción de todos los seres que habitan un territorio, incluyendo, por lo tanto, la naturaleza, sus elementos y seres vivientes 
que comparten su hábitat con los seres humanos. Por lo tanto, buen vivir y bienestar son aspectos interrelacionados para los pueblos originarios. En países como Ecuador, el concepto Sumak Kawsai [Buen Vivir] se ha asociado a la idea de bien común de la humanidad.

En Chile, una de las primeras investigaciones empíricas que se aproxima al estudio del bienestar y calidad de vida en el pueblo mapuche, advierte que "fenómenos como la relocalización y la pérdida de sus asentamientos históricos impactan en las condiciones de vida de este pueblo en su propio territorio" (Díaz, Pérez, González, \& Simon, 2004, p. 10). Sobre el particular, en lengua mapuche existen constructos que relacionan bienestar a salud/enfermedad, a sus causas, a procedimientos terapéuticos y al reconocimiento de la existencia de seres cualificados para intermediar entre la enfermedad y el retorno del equilibrio y el bienestar de la persona (Díaz et al., 2004). Con anterioridad, en la aplicación de modelos de Salud Intercultural en la Región de la Araucanía se había descrito el equilibrio entre la persona, la naturaleza y lo sobrenatural como küme mongen [vida buena o buen vivir], consignando que la mantención de este equilibrio se asocia en la cosmovisión mapuche a la idea de salud, mientras que su ruptura se vincula a la idea de enfermedad (Ibacache, 1997; Hansen, 2012).

En el pensamiento mapuche, los valores y prácticas asociadas al "estar bien” o al "estar mal", se basan en cuatro principios: che [persona]; kimün [saber]; newen [fuerza] y; küme [estar bien espiritual y materialmente]. Así, se puede distinguir el bienestar en armonía o küme felen, el bienestar en la calidad de vida o küme mongen y el bienestar otorgado por la ley natural o autorregulación de la naturaleza de las distintas dimensiones del mundo mapuche donde habitan los hombres, mujeres y las fuerzas de la vida desde tiempos inmemoriales [nor-felen] (Quilaqueo, citado en Tricot 2009). En cuanto a la relación con el entorno, se sostiene que el aire, el suelo, el subsuelo, las montañas, los ríos, los animales y los seres humanos componen el introfill mongen [biodiversidada], encontrándose todos ellos en una interrelación donde cada uno está situado en un mismo nivel, y cuyas posiciones están directamente relacionadas con el logro del equilibrio. En la medida en que se respeten los equilibrios se podría acercar al estar bien [küme fülen]. El estar consciente de la convivencia de las diferentes formas de vida que habitan los espacios, provee igualmente bienestar para quienes allí conviven (Marimán \& Aylwin, 2008). El concepto de küme mongen, o buen vivir, plantea la existencia de un vínculo entre identidad, persona, pueblo, territorio, medio ambiente, naturaleza, bienestar y desarrollo ${ }^{2}$.

A la luz de los antecedentes consignados, la pregunta de investigación que emerge en este estudio es: ¿Cuáles son los alcances de los imaginarios sociales del bienestar en personas mapuche del Gran Concepción? En consecuencia, el objetivo que guía el presente estudio es analizar los imaginarios sociales del bienestar en personas mapuche del Gran Concepción. En este sentido, la relevancia de este estudio se inscribe en la atención brindada en torno a los imaginarios sociales del bienestar en personas que se definen como mapuche y cuentan con residencia urbana, lo que además subvierte la imagen estereotipada del mapuche tradicional de contexto rural.

\section{Material y método}

La investigación que dio sustento a este artículo se desarrolló entre los años 2013 y 2016, durante los cuales se analizó material bibliográfico y se ejecutó el trabajo de campo 
etnográfico. Se aplicaron 58 entrevistas en profundidad a 36 mujeres y 22 hombres de entre 18 y 60 años, pertenecientes a los estratos socio-económicos medio y bajo, en los cuales se ubica mayoritariamente la población mapuche en Chile. Además, se realizaron 20 grupos de discusión con la participación de 145 personas, 56 hombres y 89 mujeres, pertenecientes a diferentes espacios organizacionales y sociales, conformado por sindicatos, adultos mayores, estudiantes, servidores públicos, grupos de salud, grupos de profesionales y trabajadores, organizaciones de mujeres. El criterio de selección para las personas entrevistadas fue el auto identificarse como mapuche o tener ascendencia mapuche, y en el caso de los grupos de discusión, los participantes no debían tener adscripción a un grupo étnico. A través de la aplicación de ambas técnicas se indagó en la construcción de imaginarios sociales acerca de la identidad mapuche en medios urbanos, en el Gran Concepción, Chile. En este artículo se reportan únicamente los resultados de la información relativa a las entrevistas semi-estructuradas. Las entrevistas fueron transcritas literalmente y luego codificadas de acuerdo con la región, comuna de residencia, sexo, y número secuencial. El análisis de las entrevistas se llevó a cabo una por una, como fuente de análisis primaria, por medio de análisis hermenéutico y análisis de discurso, con la ayuda del programa de análisis cualitativo Atlas-T, arrojando un conjunto de corpus discursivos que, una vez agrupados por categorías (sacos semánticos) y organizados en una malla temática, dieron origen a este artículo. Las unidades de análisis secundarias fueron párrafos u oraciones textuales de los entrevistados trabajadas a partir de la malla temática donde se organizaron los discursos en torno a las categorías de análisis central: bienestar subjetivo individual, bienestar subjetivo a nivel colectivo y bienestar subjetivo a nivel macrosocial. Todo lo anterior respetando los sentidos contextuales aportados por los hablantes. Dichos corpus dan cuenta de la manera en que las personas mapuche entrevistadas entienden el tema del bienestar y lo asocian a su identidad.

El tema del bienestar subjetivo, no considerado como una pregunta explícita en la pauta de entrevista en profundidad acerca del tema de la identidad, emergió como categoría de significado en el transcurso de la investigación, en los discursos de las personas entrevistadas que se identificaban con el pueblo mapuche. Así, puede señalarse que al indagar en las subjetividades de los actores en relación al tema de la identidad con el pueblo mapuche, emerge el tema del bienestar subjetivo asociado a la vivencia de la identidad étnica.

\section{Resultados del análisis}

\section{Imaginarios sociales del bienestar en el plano individual}

A través de las entrevistas se detectó que en personas que se reconocen como mapuche, el bienestar subjetivo se percibe principalmente cuando la identidad étnica es auto-reconocida y hetero-reconocida positivamente. En este sentido, se torna importante el conocer la cultura y vivirla, el sentirse mapuche y ser reconocido como tal por el grupo. También, las formas de expresividad cultural externalizadas son importantes, ya que provocan o refuerzan sentimientos de pertenencia al pueblo mapuche. El auto reconocimiento es considerado una fuente que potencia la autoestima; aporta felicidad y revitalización, pues otorga sentido a la vida, a nivel micro y meso social. 
Cuando conocí a otras personas mapuche (en la ciudad) me identifiqué con ellos, me sentí contenida, me sentí acompañada. $Y$ como entré a la Universidad sabía que ser diferente no era malo y empecé a buscar conocimiento, fue un proceso lento, pero mientras más aprendía más me sentía mapuche. El kimün [conocimiento] es lo que a uno le da identidad, ayudando a uno a encontrarse y sentirse feliz y con un sentido en la vida. [Entrevista 57].

No había ningún tipo de conexión de mis abuelos; mis tíos desconocian la información (acerca del ser mapuche); no hubo esa transmisión oral que perdí, pero que adquirí después de los 30 y de las mejores fuentes: con ñañas [mujeres mayores] poderosas y con lonkos [autoridad tradicional, jefes de las comunidades o reducciones] fue con quienes descubrí lo que me faltaba" [Entrevista 51 ].

La demostración de los aspectos manifiestos de la identidad son acciones simbólicas que conllevan un "sentirse bien", entre los cuales aparecen elementos objetivos como el uso de vestimentas, el conocimiento y práctica de la lengua mapuche. Por el contrario, el no saber hablar mapudungun [lengua mapuche] causa malestar, pues el idioma es la puerta de entrada a las ceremonias, a los significados, a la introspección, y se plantea que su pérdida implicaría la de los simbolismos ceremoniales, afectando también el bienestar colectivo.

"Cuando yo me pongo mi vestimenta no me visto en cualquier lado, me visto siempre en mi casa... porque uno no tiene que avergonzarse de lo que e." [Entrevista 16]

"La cosmovisión, el idioma, son fundamentales. Creo que el idioma es fundamental, de hecho con solo aprender el idioma uno comprende y eso es súper esencial para los mapuche que estamos aqui en la ciudad, porque son conexiones." [Entrevista 45]

A nivel individual el bienestar aparece asociado a la interrelación de un proceso individual y grupal, ya que las personas se identifican y se sienten parte de un colectivo lo que les refuerza su propia identidad. El posicionamiento temporal (analizando su historia presente y pasada), les permite "reconciliarse" con su pasado con el fin de vivir un presente "feliz", resignificando su identidad con "sentido de existencia" individual y colectivo.

\section{Imaginarios sociales del bienestar a nivel colectivo}

En el nivel meso social es donde se construyen las interrelaciones entre las personas y se concreta la identidad grupal, que refuerza la identidad personal y grupal a través de la acción colectiva, provocando un sentimiento positivo de pertenencia y solidaridad. El sentirse bien se manifiesta, además, en el sentido de completitud que las personas logran al descubrir, a través de los otros miembros de su colectividad, parte de la esencia mapuche, lo que les permite "reconstruir" una identidad étnica "queriéndose a sí mismos". La conexión de la familia o la organización con la comunidad y el territorio se considera importante en la construcción imaginario social de la identidad, confirmándose la idea holística de bienestar subjetivo, pues el territorio común no es sólo físico, sino que es simbólico y se encuentra asociado a la ancestralidad y la comunidad.

La gente que se siente mapuche tiene una conexión con el territorio, tiene una conexión con sus antiguos, y entre nosotros mismos ... el pueblo mapuche y todos los mapuche se 
sienten apoyados en los otros ... somos todos uno y he conocido a tantos que sin conocerlos también ellos sienten una conexión conmigo, y quiero recalcar la unión y una esencia, un sentir de todos. [Entrevista 45]

Por otro lado, el formar parte de organizaciones sociales urbanas, que fueron creadas en las ciudades a partir de la Ley Indígena (1993), es valorado positivamente en la (re) significación identitaria, al ser entidades difusoras de la cultura y donde se puede refrendar la identidad y vivirla abierta y colectivamente. En este sentido, las organizaciones validan el sentido de pertenencia a través del "rescate cultural" de las tradiciones y ceremonias. Se plantea la sincronía que hay entre el pensar y el actuar, y que se vivencia en las ceremonias, donde la comprensión que se logra en los rituales vividos genera un sentimiento de felicidad, ayudando a concretizar la idea de pertenencia a una colectividad y a un territorio. La organización se reconoce también como un vínculo entre la comunidad imaginada (rural) y el mapuche que habita en la ciudad, lo que se manifiesta además en la apropiación de espacios urbanos para la realización de sus actividades colectivas significativas.

Creo que refuerza la identidad mapuche [la organización], la va reconstruyendo o bien se va recuperando. Creo que son vitales porque existen necesidades por parte de la población por poder trabajar en comunidad lo que significa asociarse para poder trabajar el sentido de identidad. Uno puede construir la identidad mapuche a diferentes escalas pero creo que la que más te valida es la asociación, si uno trabaja por fuera es menos válido. [Entrevista 12]

"La ceremonia, me renueva; ahí se toma conciencia de lo que es ser mapuche. Hay gente, sale el sol y la energía uno la siente y es feliz" [Entrevista 58].

Para el centro cultural Lafken [mar] palife [jugador de un juego consistente en desplazar una pelota con ayuda de un bastón] la laguna de San Pedro es como una iglesia para nosotros, como la de los católico ... es un centro ceremonial mapuche que tenemos. Se hacen los nguillatunes [rogativa], los wetripantü [conmemoración del solsticio de invierno], se juega palin [juego tradicional]... tenemos el rewe [altar ceremonial]. [Entrevista 3]

\section{Imaginarios sociales del bienestar subjetivo a nivel macro social o estructural}

A nivel macro social, los imaginarios sociales del bienestar subjetivo se encuentran asociados a la relación holística que se establece con el medio. Así, si se considera que si el sistema en general está bien, la persona estará bien; pero si se entiende que el sistema está enfermo o dañado, las personas se sentirán afectadas en igual medida. En este sentido el bienestar es colectivo y abarca la relación con el entorno territorial, ambiental, económico, político, social y cultural. En este nivel, el bienestar colectivo se sitúa por sobre el bienestar individual.

Estar mejor es sentir que a mi alrededor, mi gente mapuche está, en cuanto a lo político, lo económico, lo social, lo cultural; y uno también está bien cuando el territorio está bien, cuando el medio ambiente está bien, entonces uno va a nuestra idea de küme mongen [buen vivir], donde estar bien tiene que ver con todo. Uno no puede estar bien si hay un 
hermano sufriendo, si está el megaproyecto Octopus [Construcción y operación de un terminal marítimo de gas natural licuado], instalándose en la bahía de Concepción o si el Estado está reprimiendo a la gente. [Entrevista 58]

En consideración a la historia de pérdida sistemática de los territorios donde habitaba el pueblo mapuche a la llegada de los conquistadores y colonos, y a la posterior sujeción moderna a los intereses de empresas hidroeléctricas y forestales; en la región donde se desarrolló esta investigación, los imaginarios sociales del bienestar, se construyen en sentido opuesto al deterioro y al malestar que pueda observarse en el entorno. Así, las personas entrevistadas expresan el malestar que sienten en su vivencia cotidiana, también a partir del medio social y ecológico en el que se desenvuelven, lo que impacta directamente su percepción de bienestar subjetivo. En síntesis, las percepciones acerca de lo que provoca el malestar colectivo, apuntan a distintos ámbitos de la vida social de las personas mapuche, que sienten violentada su identidad, tanto individual como colectivamente:

Malestar con el Estado: Esta dimensión comprende discursos identificados en participantes que dan cuenta de prácticas del Estado que han suscitado malestar para el pueblo mapuche.

"Legalmente no somos respetados" [Entrevista 21].

"El Estado no quiere aceptar que existe un pueblo, niega al pueblo mapuche" [Entrevista 20].

"Nos quitaron tierras y perdimos nuestra cultura" [Entrevista 32].

\section{Malestar con el municipio:}

Lo bueno es que en la municipalidad existe una oficina de pueblos originarios, pero lo malo es que cuando ya está el pan cocinado [la decisiones ya están previamente tomadas por otros], recién ahi nos llaman a nosotros, porque al final ellos trabajan aparte ... se toman muchas atribuciones, deciden por nosotros, votan por nosotros, y ahi recién, nos invitan. [Entrevista 35]

Malestar generado por proyectos de empresas: Esta dimensión se asocia a discursos identificados en participantes que exponen prácticas de empresas hidroeléctricas o forestales que han favorecido el malestar para el pueblo mapuche.

Los mismo rewe [centros ceremoniales] que son pasados a llevar por grandes proyectos, por las mismas empresas forestales, pasan a llevar mucho la cosmovisión ... el que les quiten estos terrenos, los ríos, los maltraten, les llega mucho, lo que es un daño tremendo. Hay muchas personas que les cuesta ver lo que hay detrás de un árbol, y sobre todo para los mapuche lo importante que puede ser un lugar. [Entrevista 57]

Malestar con las fuerzas policiales, violaciones a los Derechos Humanos: Esta dimensión integra discursos identificados en participantes que manifiestan directamente dinámicas de violencia ejercida de parte de la policía militarizada de Chile hacia el pueblo mapuche.

"En las comunidades los carabineros [policía militarizada chilena] se siguen metiendo, siguen atacando a los niños y eso ¡no ha cambiado!" [Entrevista 23]. 
Por otro lado, la discriminación percibida también se presenta como una fuente de malestar, lo que inhibe la vivencia del bienestar y obstaculiza el proceso de desarrollo humano, en tanto no contribuye a la conformación de la autoestima y el autoconcepto en niños y niñas mapuche. Además, desde un enfoque de salud psicobiosocial, las personas adultas que declaran sentirse víctimas de discriminación étnica, mostrarían indicadores más altos de malestar físico y emocional, asociados a sintomatología ansioso-depresiva, que el resto de la población. Esto podría ser producto de la vivencia de sentimientos negativos como la vergüenza, la desesperanza y la sensación de pérdida cultural.

"Porque hubo un momento en Chile que las personas renegaban de ser mapuche porque habia mucha discriminación, no tenían oportunidad de trabajo, ni de estudio, en los colegios se burlaban de los niños con apellido mapuche [Entrevista 28].

Cuando uno dice su apellido lo asocian con que son peleadores ... me molestan en la casa donde trabajo porque cuando en las noticias hablan de los mapuche me dicen que ahí está mi famili; y así somos discriminados por ser mapuche [Entrevista 11]

"Cuando yo empecé a crecer mi abuelo ya no hablaba en mapudungun, porque le daba vergüenza, porque éramos muy discriminados; eran los rasgos demasiados marcados y para la gente era el Indio" [Entrevista 34].

En este sentido también hay un llamado a romper con los estereotipos actuales asociados a la población mapuche:

Pienso que hay algo más bonito que rescatar y que mostrar también y en eso es lo que nosotros tenemos que trabajar, en mostrar lo bonito de la cultura y hacérselo entender a la gente y que no asimile al pueblo mapuche sólo con terrorismo, con incendios y que se entienda también la lucha [Entrevista 57].

\section{Discusión}

Diversos son los elementos que configuran los imaginarios sociales del bienestar en personas que se auto identifican como mapuche, con arreglo a los diferentes planos en que se vivencia la identidad. Las fuentes de bienestar subjetivo que emergen de estos imaginarios provienen así de los distintos niveles en que la identidad es vivida y expresada.

A nivel individual, donde se expresan los sentimientos y las vivencias personales, los imaginarios sociales del bienestar se construyen en torno a la resignificación de la identidad. En este plano son fuente de bienestar: la percepción positiva de la identidad; la percepción positiva del reconocimiento del endogrupo (mapuche); la generación de sentido de pertenencia a una colectividad mapuche (real o imaginada); el reconocimiento positivo del exogrupo (no mapuche); vivir procesos de reconciliación respecto de un pasado de no aceptación y olvido; conocer la cultura y cosmovisión mapuche; expresar la cultura a través del uso de la vestimenta, joyas y el aprendizaje y práctica del mapudungun.

A nivel colectivo, que refiere a las relaciones con el grupo y la comunidad, la idea de bienestar se vincula a la construcción de una identidad colectiva. En este plano el bienes- 
tar se asocia a: la generación de sentimientos de pertenencia y solidaridad comunes; a la participación en asociaciones indígenas; participación en acciones colectivas significativas como celebración de ceremonias y participación en juegos; traspaso de conocimientos de una generación a otra; visibilización de la identidad mapuche colectiva; conexiones identitarias con un territorio (físico y simbólico) y/o comunidad mapuche; generación de vínculos con la comunidad rural y urbana.

A nivel macro social, donde operan determinantes estructurales que provienen del entorno, el bienestar se configura como un imaginario social del malestar, en la medida en que se comparten percepciones de malestar en su relación con las esferas públicas, en la relación histórica de violencia con el Estado y con las empresas que han explotado sus tierras. Este imaginario social, que refiere a ausencia de bienestar, se fundamenta en experiencias concretas de malestar que dan cuenta de la falta de armonía a nivel social, político, cultural y ambiental; de la vivencia de discriminación por parte del Estado y de la sociedad; de la violencia que emana por el no reconocimiento de los pueblos indígenas en la Constitución; de la violencia institucional observada en las fuerzas policiales en las comunidades mapuche; de la ausencia de participación mapuche en el diseño de planes o políticas sociales en los gobiernos locales y municipios; de la pérdida de territorio mapuche; de la pérdida de la cultura mapuche; de un sistema económico productivo que se contrapone a la cosmovisión mapuche; a la existencia de estereotipos y estigmatizaciones hacia el pueblo mapuche.

Desde el dominio de comprensión que se configura en torno a estos resultados, los imaginarios de la población mapuche en torno al bienestar se distancian de una concepción economicista y materialista del mismo asociado a su cuantificación y medición como variable, por el contrario, se orientó al plano de las significaciones. Es decir, para las personas mapuche, el bienestar se construye de manera socio imaginaria como parte de un proceso de relación entre sujetos, entorno y/o naturaleza, asociado a las condiciones que permiten sostenerlo o transformarlo, y en su construcción confluyen percepciones, representaciones, valoraciones y emociones asociadas no solo a la felicidad, la seguridad y el respeto, sino también a la trascendencia como pueblo portador de una identidad diferenciada. Así, los hallazgos muestran que en los niveles individual o grupal el imaginario en torno a la identidad se configura como presencia de bienestar, en el sentido de "Buen Vivir", o estar bien con la naturaleza y el entorno social. Ello contrasta con el nivel macrosocial o de la estructura de la sociedad, en el cual el imaginario social que vincula la relación del pueblo mapuche con el Estado de Chile no solamente refiere a la ausencia de dicho bienestar sino que a la presencia de malestar. En consecuencia, el haber integrado la noción de "Buen Vivir", permitió al estudio ser sensible a la particularidad de este en los participantes, involucrando la identidad, territorio, bienestar y desarrollo, entre otros. En relación a ello, se presenta la paradoja de que si bien se reconoce un bienestar en los niveles individual y colectivo de vivencia de la identidad mapuche, éste se ve alterado por el contexto socio-histórico, político y económico en torno a los pueblos originarios y al reconocimiento de sus derechos, que afectan sus condiciones y posibilidades de existencia. Dicho de otra manera el bienestar vivenciado a nivel individual y colectivo no es percibido de la misma manera a nivel estructural o macrosocial, donde la identidad se ve impactada por condiciones estructurales que los afectan como pueblo. Estas condiciones determinan a los mapuche en particular y a los pueblos indígenas en general a situaciones de menores ingresos, menor acceso a la salud, menor cobertura educacional y mayor pérdida de la lengua 
materna y a evidenciar indicadores de mayor marginalidad social y económica (PNUD, 2013).Finalmente, cabe señalar que este estudio no se encuentra exento de limitaciones. En primer lugar, no se realizó un análisis de los discursos de los participantes mapuche según tiempo de permanencia en la zona, o si eran oriundos urbanos, ni tampoco se distinguió eventuales pertenencias o vínculos con agrupaciones ambientalistas. En segundo lugar, los resultados de esta investigación dan cuenta de una versión asociada al encuentro entre participantes de esta investigación y el equipo de investigación, por lo que los resultados no necesariamente se extrapolan a la realidad de todo el pueblo mapuche urbano de la ciudad de Concepción, o de otras ciudades del país y tampoco, al pueblo mapuche rural. De acuerdo con lo consignado, los resultados deben procurar ser considerados con cautela y precisión. Es de gran relevancia que futuras investigaciones profundicen el estudio del bienestar, y en específico en pueblos indígenas u otros sectores de la sociedad que sistemática e históricamente han sido discriminados o negados. Se estima que un enfoque cualitativo e interdisciplinario en psicología, puede brindar gran soporte en tal tarea.

\section{Notas}

${ }^{1}$ La costumbre local exige que el plural de mapuche se escriba sin letra s. Lo anterior, apelando a que la construcción de la palabra - mapu [tierra], che [gente]-determina la pluralidad en sí, por lo que la construcción lingüística mapuches con el morfema de número sería una redundancia. De todas maneras, es común que en español de América del Sur no se use el plural del nombre ni del adjetivo de un pueblo originario (Aravena \& Jara, 2016).

${ }^{2}$ Véase por ejemplo el informe del PNUD sobre los aspectos metodológicos de generar un índice de desarrollo humano en población mapuche (PNUD, 2013)

\section{Referencias}

Acosta, A. \& Martínez, E. (2009). El buen vivir. Una vía para el desarrollo. Quito: Abya Yala. Antilleo, E. (2010). Urbano e indígena: dialogo y reflexión en Santiago Warria. Working Paper Series 31. Gothenberg: Nuke Mapuforlaget. Recuperado de http://www. mapuche.info/mapuint/antileo20100919.pdf

Aravena, A. (1995). Desarrollo y procesos identitarios en el mundo indígena urbano. In Tierra, Territorio y Desarrollo Indígena (pp. 171-178). Temuco, Chile: Instituto de Estudios Indígenas de la Universidad de la Frontera.

Aravena, A. (2001). Shadows in the big city. El Correo de la UNESCO, Dossier Colour, nation, ethnic hate: Why racism? 54(9), 18-19.

Aravena, A. (2002). Los Mapuche - Warriache: Procesos migratorios contemporáneos e identidad mapuche urbana. In G. Boccara (Coord.), Colonizacion, resistencia y mestizaje en las Américas, Siglos XVI-XX (pp. 359-385). Quito: Abya-Yala.

Aravena, A. (2003). El rol de las memorias individuales y de la memoria colectiva en la conversión identitaria de los mapuches en Santiago. Revista de Estudios Atacameños 26, 89-96.

Aravena, A. (2006). Identidades étnicas, identidades sociales. La etnicidad de cara al siglo XXI. Proposiciones, 35, 336-347. 
Aravena, A. \& Baeza, M. (2017). Imaginarios sociales y construcción intersubjetiva de alteridad. La prensa escrita y la cuestión mapuche en Chile. Cultura y representaciones soc. Recuperado de http://www.scielo.org.mx/scielo.php?script=sci abstract\&pid=S2007$\underline{81102017000200007 \& \operatorname{lng}=\text { es\&nrm=iso. }}$

Aravena, A. \& Cerda, C. (2018). Indígenas urbanos en Chile: imaginarios sociales de la identidad mapuche en la frontera del Biobío. In J. E. Horbath \& M. A. Gracia (Coords.), La cuestión indígena en las ciudades de las Américas Procesos, políticas e identidades (pp. 335374). Clacso/Conacyt/Ecosur. Buenos Aires: Miño y Dávila Editores.

Aravena, A. \& Jara, F. (2016). Antropologia jurídica y superposición de sistemas normativos Estado/nación - pueblos indígenas: el caso actual del pueblo Mapuche. AIBR, Revista de Antropología Iberoamericana, 11(03), 319-340. DOI:10.11156/aibr.110302

Baeza, M. (2000). Los caminos invisibles de la realidad social: ensayo de sociología profunda sobre los imaginarios sociales. Chile: Ril Editores.

Baeza, M. (2015). Hacer mundo. Significaciones imaginario-sociales para construir sociedad. Chile: Ril Editores.

Bartolomé, M. (2008). Procesos interculturales. Antropología política del pluralismo cultural en América Latina. México: Editorial Siglo XXI.

Bengoa, J. (1985). Historia del pueblo mapuche: siglos XIX y XX. Santiago: Ediciones Sur.

Bengoa, J. (1990). Breve historia de la legislación indígena en Chile. Santiago: Comisión Especial de Pueblos Indígenas.

Berger, P. \& Luckmann, T. (1968). La construcción social de la realidad. Buenos Aires: Amorrortu.

Boccara, G. \& Seguel-Boccara, I. (1999). Políticas indígenas en Chile (siglos XIX , XX): de la asimilación al pluralismo. El caso mapuche. Revista de Indias, LIX (217), 742-774.

Bolados, P. (2012). Neoliberalismo multicultural en el Chile postdictadura: la política indígena en salud y sus efectos en comunidades mapuches y atacameñas. Chungara, 44(1), 135-144.

Callbucura, J. (2006). Nación Mapuche. Contrapunto conceptual. Chile: Jorge Calbucura.

Carretero, Á. (2001). Imaginarios sociales y crítica ideológica. Una perspectiva para la comprensión de la legitimación del orden social. Alicante: Biblioteca Virtual Miguel de Cervantes. Recuperado de http://www.cervantesvirtual.com/obra/imaginarios-sociales-y-critica-ideologi$\underline{\mathrm{ca}-\mathrm{-}-\mathrm{l}}$

Castoriadis, C. (2007). L'imaginaire comme tel. Paris: Hermann Éditeurs.

De Rivera, J., Kurrien, R., \& Olsen, N. (2007). The emotional climate of nations and their culture of peace. Journal of Social Issues, 63(2), 255-271. doi: 10.1111/j.1540-4560.2007.00507.x Díaz, A., Pérez, M., González, C., y Simon, J. (2004). Conceptos de enfermedad y sanación en la cosmovisión mapuche e impacto de la cultura occidental. Ciencia y Enfermería, 10(1), 9-16. Recuperado de http://dx.doi.org/10.4067/S0717-95532004000100002

Diener, E., Suh, E., Lucas, R., \& Smith, H. (1999). Subjective well-being: three decades of progress. Psychological Bulletin, 125(2), 276-302. doi: 10.1037/0033-2909.125.2.276

Elcheroth, G., Doise, W., \& Reicher, S. (2011). On the knowledge of politics and the politics of knowledge: how a social representations approach helps us rethink the subject of political psychology. Political Psychology, 32(5), 729-758. doi:10.1111/j.1467-9221.2011.00834.x

Erickson, E. (1971). Identidad, juventud y crisis. Buenos Aires: Paidós.

Espinosa, A., \& Tapia, G. (2011). Identidad nacional como fuente de bienestar subjetivo y social. Boletín de Psicología, 102, 71-87. Recuperado de http://www.uv.es/seoane/boletin/previos/N102-5.pdf

Farías, F., Orellana, C., y Pérez, C. (2015). Perfil de las publicaciones sobre bienestar subjetivo en Chile. Cinta de Moebio. 54, 240-249. Recuperado desde www.moebio.uchile.cl/54/farias. $\underline{\mathrm{html}}$ 
Gergen, K. (1996). La crisis de la representación y la emergencia de la construcción social. In Realidades y relaciones. Aproximaciones a la construcción social (pp. 29-57). Barcelona: Paidos.

Goicovich, F. (2007). Entre la conquista y la consolidación fronteriza: dispositivos de poder hispánicos en los bosques meridionales del reino de Chile durante la etapa de transición (15981683). Historia, 40(2), 311-332.

González, H. (1986). Propiedad comunitaria o individual: las leyes indígenas y el pueblo mapuche. Nütram, III (3), 7-13.

Gudynas, E. (2009), El mandato ecológico. Derechos de la naturaleza y políticas ambientales en la nueva constitución. Quito: Abya Yala.

Hasen, F. (2012). Interculturalidad en salud: competencias en prácticas de salud con población indígena. Ciencia y Enfermería, 18(3), 17-24.

Ilbacache, J. (1997). La salud, el desarrollo y la equidad en un contexto intercultural. Recuperado de http://www.mapuche.info/mapuint/ibaca00.htm.

Ilbáñez, T. (2001). Construccionismo y psicología. Municiones para disidentes. Barcelona: Gedisa.

Ilbáñez, T. (2003). La construcción social del socioconstruccionismo: retrospectiva y perspectivas. Política y Sociedad, 40(1)155-160.

Instituto Nacional de Derechos Humanos - INDH. (2017). Informe anual situación de los Derechos en Chile. Santiago: Autor

Larrea, A. (2010). La disputa de sentidos por el buen vivir como proceso contrahegemónico. En memorias Secretaría Nacional de Planificación y Desarrollo (comp.). Los nuevos retos de América Latina. socialismo y sumak kawsay (pp. 15-27). Ecuador: Senplades.

Ley Indígena. (1993). Nueva ley indígena. Santiago: Gobierno de Chile.

Marimán, P. \& Aylwin, J. (2008). Las identidades territoriales mapuche y el Estado de chileno: conflicto interétnico en un contexto de globalización. In X. Leyva, A. Burguete, \& S. Speed (Coords.), Gobernar (en) la diversidad: experiencias indígenas desde América Latina. Hacia la investigación de co-labor (pp. 111-150). México: Publicaciones de la Casa Chata.

Merino, M. \& Tocornal, X. (2012). Posicionamientos discursivos en la construcción de identidad étnica en adolescentes mapuches de Temuco y Santiago. Revista Signos, 45(79), 154-175.

Merino, M., Quillaqueo, D., \& Sáiz, J. (2008). Una tipología del discurso de discriminación percibida en mapuches de Chile. Revista Signos, 41(67), 279-297.

Merino, M. (2007). El discurso de la discriminación percibida en mapuches en Chile. Universidad Católica de Temuco, Chile. Discurso \& Sociedad, 1(4), 604-622.

Ministerio de Desarrollo Social. (2015). CASEN 2013. Pueblos indígenas. Síntesis de resultados. Recuperado de http://observatorio.ministeriodesarrollosocial.gob.cl/documentos/ Casen2013 Pueblos Indigenas 13 mar15 publicacion.pdf

Montero, M. (2004). Introducción a la psicología comunitaria. Desarrollo, conceptos y procesos. Buenos Aires: Paidós.

Moulian, T. \& Marín, G. (1998). El consumo me consume. Santiago: Lom

Mujica, L. (2007). Hacia la formación de las identidades. In J. Ansión \& F. Turbino (Eds.), Educar en ciudadanía intercultural (pp. 11-36). Temuco, Chile: Fondo Editorial.

Ortiz, C. (2015). El Parlamento de Quilin del año 1641: una aproximación a las relaciones interlinajes a partir de la vida fronteriza. Cuadernos de Historia, 42, 7-31.

Páez, D., Martín-Beristain, C., González, J., Basabe, N., \& De Rivera, J. (Eds). (2011). Superando la violencia colectiva y construyendo cultura de paz. Madrid: Editorial Fundamentos. Páez, D., Ruiz, J., Gailly, O., Kornblit, A., Wiesenfeld, E., \& Vidal, C. (1997). Clima emocional: su concepto y medición mediante una investigación transcultural. Revista de Psicología Social, 12(1) 79-98. 
Pairican, F. (2014). Malon. La rebelión del movimiento mapuche 1990-2013. Santiago: Pehuén Editores.

Paramo, P. (2008). Psychosocial construction of identity and self. Revista Latinoamericana de Psicología, 40(3), 539-550.

Phinney, J. \& Ong, A. (2007). Conceptualization and measuremente of ethnic identity. International Journal of Behavioral Development, 31(5), 478-490.

Programa de las Naciones Unidas para el Desarrollo - PNUD. (2012). Desarrollo humano en Chile. Bienestar subjetivo: el desafio de repensar el desarrollo. Santiago de Chile. Recuperado de http://desarrollohumano.cl/idh/informes/2012-bienestar-subjetivo-el-desafio-derepensar-el-desarrollo/

Programa de las Naciones Unidas para el Desarrollo - PNUD. (2013). Índice de Desarrollo Humano en la Población Mapuche, 2013.

Tajfel, H. (1981). Grupos humanos y categorías sociales: estudios de psicología social. Barcelona: Helder.

Taylor, C. (2006). Imaginarios sociales modernos. Barcelona: Paidós Ibérica.

Tricot, T. (2009). El nuevo movimiento mapuche: hacia la (re)construcción del mundo y el país mapuche. Polis (Santiago), 8(24), 175-196.

Turmer, J. (1985). Social categorization and the self-concept: A social cognitive theory of group behaviour. In E. J. Lawler (Ed.), Advances in group processes: Theory and research (Vol., 2, pp. 77-122). Greenwich: JAI Press.

Vanhulst, J. (2015). El laberinto de los discursos del buen vivir: entre sumak kawsay y socialismo del siglo XXI. Polis. 40. doi: 10.4000/polis.10727

Vygotsky, L. (1996). Psicología infantil. Obras escogidas (Vol. 4). Madrid: Visor.

Verkuyten, M. \& Wolf, A. (2002). Being, feeling and doing: discourses and ethnic self-definitions among minority group members. Culture and Psichology, 8(4), 271-399.

\section{ANDREA ARAVENA REYES \\ https://orcid.org/OOOO-0001-7112-3794}

Antropóloga, doctora en Antropología social y etnología por la Escuela de Altos Estudios en Ciencias Sociales de París. Académica Facultad de Ciencias Sociales, Universidad de Concepción, Chile.

Endereço institucional: Facultad de Ciencias Sociales - Universidad de Concepción, Bío Bío,

CHILE.

E-mail: andrea.aravena@,udec.cl

\section{CLAUDIA CERDA ZÚÑIGA \\ https://orcid.org/0000-0001-9478-9978}

Licenciada en Sociología Universidad de Chile; magíster en Investigación Social y Desarrollo por la Universidad de Concepción; Diplomada en Gestión en Políticas Indígenas e Interculturalidad. Universidad del Desarrollo, Chile.

E-mail: claudiaxcz@gmail.com 
Psicólogo político y Psicoanalista por la Universidad Andrés Bello, Chile. Magíster en Psicología Social. Magíster en Psicología Clínica. Doctor en Psicología, Universidad de Concepción, Chile. Académico Facultad de Educación y Humanidades Universidad del Bío- Bío, Chile. Becario Conicyt, Chile. Becario Fundación Volcán Calbuco, Chile.

E-mail:p.alcota@gmail.com

\section{NATALIA ZAÑARTU CANIHUANTE}

https://orcid.org/0000-0003-3434-9721

Psicóloga por la Universidad de la Frontera, Chile. Doctora en Psicología por la Universidad de Concepción, Chile. Filiación a Facultad de Psicología, Universidad San Sebastián, Concepción, Chile.

E-mail: natalia.zanartu@docente.uss.cl

\begin{tabular}{|l|l|}
\hline Historial & $\begin{array}{l}\text { Fecha de recepción: 26/10/2017 } \\
\text { Fecha de revisión: 31/5/2019 } \\
\text { Fecha de aprobación:14/11/2019 }\end{array}$ \\
\hline Contribuición de & $\begin{array}{l}\text { Concepción: AAR; CCZ; NZC; PAP } \\
\text { Recolección de datos: AAR; CCZ; NZC; NMM } \\
\text { Análisis de datos: AAR; CCZ; NZC; PAP. } \\
\text { los autores }\end{array}$ \\
$\begin{array}{l}\text { Redacción del manuscrito: AAR; CCZ; PAP; NZC } \\
\text { Aprobación final del manuscrito: AAR; CCZ; PAP; NZC }\end{array}$ \\
\hline Financiación & $\begin{array}{l}\text { Artículo elaborado en el marco del Proyecto Fondecyt Iniciación } \\
11130384, \text { Chile, cuya investigadora responsable fue Andrea Ara- } \\
\text { vena Reyes. Se agradece el financiamiento a CONICYT-Chile. }\end{array}$ \\
\hline $\begin{array}{l}\text { Consentimiento de } \\
\text { uso de imagem }\end{array}$ & No se aplica \\
\hline
\end{tabular}

